\title{
CAN LEARNING OBJECTS BE REUSED - AND HOW?
}

Lessons from ARIADNE

Erik Duval

Katholieke Universiteit Leuven, http://www.cs.kuleuven.ac.be/ erikd

Abstract: This presentation will outline how many of the current efforts to work with learning objects are misguided and will present some alternative approaches that will make it possible to realize the goal of "share and reuse" that the ARIADNE Foundation has been pursuing for more than 8 years.

Key words: learning objects, metadata, standardization, ARIADNE

\section{WE'VE COME A LONG WAY}

Over the past 8 years or so, we have made solid progress in developing some of the basic standards and specifications that are needed in order to realize an open infrastructure for learning, where components from different sources can interoperate more or less seamlessly (Duval, 2004):

- the Learning Object Metadata standard enables the description of learning objects in an interchangeable way;

- the content packaging specification allows us to aggregate several learning objects together;

- content sequencing can be used to express navigational structures over the components in a package;

- through the Course Managed Instruction specification, learning objects can exchange data with Learning Management Systems,

- etc.

The SCORM reference model clarifies how these specifications and standards can work together in a coherent way. Most importantly, this set of 
basic agreements is broadly accepted, so that a state of de facto standardization is achieved.

Thus, components (authoring tools, content, management systems, instructional designs, etc.) from different vendors or non-commercial developers can work together, so that we can realize the open infrastructure mentioned above, rather than developing isolated technology islands from scratch every time anew, as used to be the case until all too recently.

\section{WE HAVE A LONG WAY TO GO}

However, it is clear that we still have a large number of issues to tackle in more advanced ways than most developers do at this moment if we want to realize the full potential of "share and reuse" as we've labeled it in the ARIADNE Foundation (Ariadne, 2004; Duval and Hodgins, 2003).

\subsection{We need more standards}

Many of the basic agreements for interoperability are at the moment not finalized standards: rather, they have the status of specifications, and, as such, cannot be guaranteed to remain available for the long periods of time that are required - remember that the TCP/IP RFC (the basic specification underlying the Internet) dates from 1981 !

ADL, the organization that maintains the SCORM model, has committed to rely only on specifications that are either open standards, or on their way to becoming such standards. This is very important, as it enforces an open and fair process in the definition and an ongoing commitment to maintenance of the basic technical definitions that underpin the global learning infrastructure.

At the moment, only the IEEE LTSC Learning Object Metadata (LOM) standard is a fully finalized standard. Even for LOM, the binding in XML is not final yet. Besides metadata, the core specifications for sequencing, packaging, repository interaction, etc. are at various points in their lifecycle.

It is vital that we maintain momentum in the development of these standards, and that we embed them in more systematic support for the communities that adopt them, in the form of good practice guidelines and application profiles.

Also, it is important that we clarify what exactly are the specific requirements on the technical level for learning applications and that we rely on more general standards where appropriate, i.e. when there are no specific requirements. At the very least, we should develop interoperability gateways between standards for learning technology and other, more generic standards 
where appropriate: for instance, how does packaging relate to MPEG-21, how does sequencing relate to SMIL, etc.

\subsection{We need better tools}

It is extremely important to understand that standards are meant to enable developers to realize interoperable technical components: standards are not meant to be visible to end users! It is understandable that early implementations of new standards and specifications focus on the implementation of the functionality required. However, it is obvious that evaluations of the actual experience of end users with these tools will show the failure of this approach.

Indeed, just like web browsers do not disclose the hairy details of HTML or HTTP, sophisticated tools should not expose for instance detailed Learning Object Metadata. We should hide those details and develop tools that do not unnecessarily burden or complicate the life of the end user. I have launched the slogan that "electronic forms must die!" in order to encourage the development of tools and infrastructure that fit well with the workflow of the end user.

As an example, in Leuven, we have integrated the ARIADNE learning object repository, called the "Knowledge Pool System", with the Blackboard Learning Management System, and we have been able to capture a detailed set of Learning Object Metadata, without requiring the end user to provide these metadata manually! Rather, we mine the data already present in the administrative system of the university, we exploit the context of operation and information about the user, etc. to deduce all the relevant data automatically behind the scenes. We believe that much more work along these lines is urgently needed, and we predict that, if such work is not undertaken, practitioners in the field will start to quickly lose interest in applying standards in reality!

Actually, we need to take this line of research much further. Our team has started to work on information visualization approaches as a radically different way of enabling access to relevant learning objects (Klerkx, J., Duval, E., and Meire, M., 2004). Similarly, social recommending techniques may help to suggest appropriate resources at the right time. Newer technologies for content syndication, like RSS, could be applied in this context as well. The overall goal is to provide flexible access to advanced functionalities for end users, without putting any additional burden on their side. 


\subsection{We need to go beyond content}

Much of the work on interoperable learning technologies has focused up to now on content and the role it plays to "deliver" learning. This was for sure the first area that needed our attention, as interoperable learning content is a basic requirement for large-scale deployment.

However, it is now time to look beyond basic content building blocks and to consider for instance how content can be "glued together" at a higher level of abstraction that current packaging and sequencing approaches allow for. It is possible that, in doing so, we will start to capture some of the instructional design aspects that are supposed to enable us to realize quality learning.

Moreover, we also need to be more serious about addressing the social aspects of learning. This includes collaborative learning support, but is much broader than that: indeed, almost all learning includes important social dynamics, and we may be able to support that aspect in a far better way, both on a personal level, as well as on that of the organization(s) that the individual belongs to.

Finally, there are important emotional aspects to learning: these relate to the social aspects already mentioned, but also include facets that are directly linked to the experience of beauty and the construction of meaning. Little attention has been spent so far on how we can provide more advanced support in this domain through technology.

\section{RECONSIDERING LEARNING}

In fact, if we consider our research field more seriously, it seems like we may have a more fundamental problem... It would be hard to argue that we have made the same sort of progress that has been made in such diverse fields as medicine, physics, biology, etc. In fact, it is not even clear that we would have consensus within the field on how to measure "progress": is the number of publications or PhD's a good indicator? Or the budgets spent, or the profits made? Where is the "learning" and how do we know that we have improved it through our efforts?

In the ProLearn Network of Excellence, I am coordinating work on precisely these questions, and on the "grand challenges" that we will need to tackle in research if we want to understand better what it is we are trying to achieve and how we can distinguish real progress from "delta research" and fashionable statements-with-all-todays-buzzwords-in-them (Prolearn, 2004). Only in this way can we get better at getting better. 


\section{CONCLUSION}

In conclusion, I believe that we have made substantial progress in the realization of an open global technical infrastructure for learning. Further work is needed in this area, and we must also shift our attention to usability issues that will determine how well our results can be put to practice. Moreover, we need to enlarge our scope to also include aspects that have been neglected so far. This will not be a small undertaking, but the potential benefits are huge: the result could be better learning for everyone (globally really!) at any time (24/7 - really!).

\section{ACKNOWLEDGEMENT}

We gratefully acknowledge the financial support of the K.U.Leuven Research Fund, in the context of the BALO project on "BAsic research on Learning Objects".

\section{REFERENCES}

Ariadne, 2004 (May 2, 2004); http://www.ariadne-eu.org/.

Duval, E., 2004, Learning technology standardization: making sense of it all, International Journal on Computer Science and Information Systems, 1(1):33-43; http://www.comsis.fon.bg.ac.yu/ComSISpdf/Volume01/InvitedPapers/ErikDuval.htm.

Duval, E., and Hodgins, W., 2003, A LOM research agenda, Proceedings of WWW2003 Twelfth International World Wide Web Conference; http://www2003.org/cdrom/papers/alternate/P659/p659-duval.html

Klerkx, J., Duval, E., and Meire, M., 2004, Using Information Visualization for Accessing Learning Object Repositories, Proceedings of IV04 - 8th International Conference on Information Visualization.

ProLearn, 2004 (May 2, 2004); http://www.prolearn-project.org/. 